\title{
Allogeneic Umbilical Cord Blood-derived HSPCS NLA101
}

National Cancer Institute

\section{Source}

National Cancer Institute. Allogeneic Umbilical Cord Blood-derived HSPCS NLA101. NCI

Thesaurus. Code C146992.

A preparation of universal, off-the-shelf, ex vivo expanded, allogeneic umbilical cord blood (UCB)-derived hematopoietic stem and progenitor cells (HSPCS) that can potentially be used to improve hematopoietic recovery after chemotherapy or hematopoietic cell transplantation (HCT). Upon administration of allogeneic HSPCS NLA101, these cells increase and restore the number of HSPCs, which may prevent or decrease infection and other complications of chemotherapy-induced neutropenia (CIN) or cord blood transplantation. 